\title{
Veröffentlichungen von Gerhard Taddey
}

\author{
Zusammengestellt von GERHARD FRITZ ${ }^{1}$
}

Die Erstellung einer Publikationsliste von Gerhard Taddey hat sich als schwierig erwiesen. Er selbst hat nur eine seit langem nicht mehr aktualisierte, längst veraltete Liste hinterlassen. Die Angaben in der baden-württembergischen Landesbibliographie erwiesen sich als ganz unvollständig, so dass an verschiedenen Stellen mühsam nachgesucht werden musste. Insofern kann es sein, dass in der nachfolgenden Liste einzelne Veröffentlichungen entgangen sind.

Abkürzungen:

BtrLK Beiträge zur Landeskunde

FoWFr Forschungen aus Württembergisch Franken

RepStAL Repertorien Staatsarchiv Ludwigsburg

VKgLKBW Veröffentlichungen der Kommission für geschichtliche Landeskunde in Baden-Württemberg

WDGB Würzburger Diözesangeschichtsblätter

WFr Württembergisch Franken

ZWLG Zeitschrift für württembergische Landesgeschichte

\section{Selbstständige Publikationen und Herausgeberschaften}

Das Kloster Heiningen von der Gründung bis zur Aufhebung. Göttingen (= Veröffentlichungen des Max-Planck-Instituts für Geschichte. Studien zur Germania Sacra 41)

Gemeinsam mit Franz Hundsnurscher: Die jüdischen Gemeinden in Baden: Denkmale, Geschichte, Schicksale. Stuttgart 1968 (= Veröffentlichungen der staatlichen Archivverwaltung Baden-Württemberg 19)

Findbuch über das Stadtarchiv Niederstotzingen 1970

In Zusammenarbeit mit Kraft Fürst zu Hohenlohe-Oehringen und Ernst Diem: Die Hospitalstiftung in Öhringen: Ein geschichtlicher Überblick. Öhringen 1976

Lexikon der deutschen Geschichte. Stuttgart 1977 (unveränd. Nachdruck 1979)

Handbuch der historischen Stätten Deutschlands VI: Baden-Württemberg. Stuttgart $^{2} 1980$

1 Gedankt sei Dorothea Bader, Ludwigsburg, und Ulrich Schludi, Neuenstein, für ihre Materialien und Hinweise. 
Lexikon der deutschen Geschichte. Stuttgart, 2. überarb. Aufl. 1983

Bearb.: So war es: Leben und Schicksal eines jüdischen Emigranten; eine Autobiographie. Von Bruno Stern. Sigmaringen 1985 (= FoWFr 23)

Findbücher über das Schloßarchiv Morstein. 5 Bde. 1985

Bearb. zus. mit Gabriele Benning: Der Deutsche Orden und das Reich: Akten der Deutschordensregierung Mergentheim; Inventar des Bestandes B 290 im Staatsarchiv. Marburg 1986 (= Beiträge zur Geschichte des Deutschen Ordens 1), S. 181-294

Red. und Mitverfasser: Bearb. v. Fritz Kempt: Pfedelbach 1037-1987. Sigmaringen 1987 (= FoWFr 30)

Hg.: Öhringen: Stadt und Stift. Öhringen 1988 (= FoWFr 31)

Kostbarkeiten aus dem Hohenlohe-Museum. Stuttgart, Korb 1988

(Red. und Hg.): Lebensbilder aus Schwaben und Franken XVII. Stuttgart 1991

Hermersberg: Die Geschichte von Schloß und Wildfuhr. Sigmaringen 1992 (= FoWFr 41)

Kein kleines Jerusalem. Sigmaringen 1992 (= FoWFr 36)

(Red. und Hg.): Lebensbilder aus Baden-Württemberg XVIII. Stuttgart 1994

Bearb. zus. mit Erwin Biemann und Wolfgang Schmierer: Israelitische Oberkirchenbehörde im Königreich Württemberg: Inventar des Bestands E $212 \mathrm{im}$ Staatsarchiv Ludwigsburg. Stuttgart 1996 (= Werkhefte der Staatlichen Archivverwaltung Baden-Württemberg, Serie C 2)

Bearb.: Kreisgerichtshof - Landgericht Heilbronn, Zivilkammer (1865-1868) 1872, 1904-1909. Ludwigsburg 1997 (= RepStAL, Bestand E 317)

Bearb. zus. mit Norbert Stein: Obertribunal Stuttgart, Kriminalsenat: 1814, 1817-1853, 1869. Ludwigsburg 1997 (= RepStAL, Bestand E 310)

Hg.: Carlo Schmid - Mitgestalter der Nachkriegsentwicklung im deutschen Südwesten: Symposium anläßlich seines 100. Geburtstages am 7.12.1996 in Mannheim. Stuttgart 1997 (=VKgLKBW, Reihe B, Forschungen, 138)

Lexikon der deutschen Geschichte. Stuttgart, 3. überarb. Aufl. 1998

(Red. und Hg.): Lebensbilder aus Baden-Württemberg XIX. Stuttgart 1998

Ministerialabteilung für den Straßen- und Wasserbau II: Bauamt für das öffentliche Wasserversorgungswesen, Kraftfahrlinien, Privat- und Nebenbahnen, 1835-1942. Bearb. v. Siegfried Schirm unter der Leitung von Gerhard Taddey. Ludwigburg 1998 (= RepStAL, Bestand E 167)

Württembergische Kommission für Landesgeschichte 1888-1954. Ludwigsburg 1999 (= RepStAL, Bestand E 216)

Hg.: Gebhard Müller: Ein Leben für das Recht und die Politik. Symposium anläßlich seines 100. Geburtstags am 17.4.2000 in Stuttgart. Stuttgart 2000 (= VKgLKBW, Reihe B, Forschungen, 148)

(Red. und Hg. gemeinsam mit Joachim Fischer): Lebensbilder aus Baden-Württemberg XX. Stuttgart 2001

(Schriftleitung gemeinsam mit Gerhard Fritz unter Mitarbeit von Herta Beutter, 
Herbert Kohl und Armin Panter): WFr 88 (2004) [Jahrbuch des Historischen Vereins für Württembergisch Franken]

Hg.: ...geschützt, geduldet, gleichberechtigt...: Die Juden im baden-württembergischen Franken vom 17. Jahrhundert bis zum Ende des Kaiserreichs (1918) [Tagung im Kloster Schöntal 29.5.-1.6.2003]. Ostfildern 2005 (= FoWFr 52)

(Schriftleitung gemeinsam mit Gerhard Fritz unter Mitarbeit von Herta Beutter, Herbert Kohl und Armin Panter): WFr 89 (2005) [Jahrbuch des Historischen Vereins für Württembergisch Franken]

(Red. und Hg.): Lebensbilder aus Baden-Württemberg XXI. Stuttgart 2005

(Schriftleitung gemeinsam mit Gerhard Fritz, Herta Beutter, Herbert Kohl und Armin Panter): WFr 90/91 (2006/2007) [Jahrbuch des Historischen Vereins für Württembergisch Franken]

Hg.: Anton Schindling: 1806 - Souveränität für Baden und Württemberg, Beginn der Modernisierung? 2007 (= VKgLKBW, Reihe B, Forschungen,169)

(Red. und Hg. zusammen mit Rainer Brüning): Lebensbilder aus Baden-Württemberg XXII. Stuttgart 2007

(Schriftleitung gemeinsam mit Gerhard Fritz, Herta Beutter, Herbert Kohl und Armin Panter): WFr 92 (2008) [Jahrbuch des Historischen Vereins für Württembergisch Franken]

(Schriftleitung gemeinsam mit Gerhard Fritz, Herta Beutter, Herbert Kohl und Armin Panter): WFr 93 (2009) [Jahrbuch des Historischen Vereins für Württembergisch Franken]

(Red. und Hg. zusammen mit Rainer Brüning): Lebensbilder aus Baden-Württemberg XXIII. Stuttgart 2010

(Schriftleitung gemeinsam mit Gerhard Fritz, Herta Beutter, Herbert Kohl und Armin Panter): WFr 94 (2010) [Jahrbuch des Historischen Vereins für Württembergisch Franken]

(Schriftleitung gemeinsam mit Gerhard Fritz, Herta Beutter, Herbert Kohl und Armin Panter): WFr 95 (2011) [Jahrbuch des Historischen Vereins für Württembergisch Franken]

(Schriftleitung gemeinsam mit Gerhard Fritz, Herta Beutter, Herbert Kohl und Armin Panter): WFr 96 (2012) [Jahrbuch des Historischen Vereins für Württembergisch Franken]

(Schriftleitung gemeinsam mit Gerhard Fritz, Herta Beutter, Herbert Kohl und Armin Panter): WFr 97 (2013) [Jahrbuch des Historischen Vereins für Württembergisch Franken]

\section{Aufsätze in Zeitschriften und in Sammelbänden}

Die jüdischen Gemeinden in Baden. Denkmale - Geschichte - Schicksale. In: BtrLK 1968, 4 
Das gemeinschaftliche Archiv in Schwäbisch Hall. In: ZWLG 28 (1969), S. 391419

Georg Herwegh und Württemberg. In: ZWLG 29 (1970), S. 190-212

Württemberger in Baden 1848. Die militärischen Operationen beim Aufstand Hecker. In: ZWLG 31 (1972), S. 312-345

Zur Biographie des Märzministers Römer. In: ZWLG 31 (1972), S. 361-366

Das Hohenlohe-Zentralarchiv. In: BtrLK 1972, 6

Ozendorf - eine Wüstungskommune in Hohenlohe. In: WFr 57 (1973), S. 86100

Wie die Hohenlohe Grafen wurden: der Erbstreit um Ziegenhain und Nidda 1450-1495. In: BtrLK 1976, 5, S. 1-9

Ernstfeuerwerkerei: Raketenversuche in Württemberg. In: ZWLG 36 (1977), S. $132-150$

Macht und Recht im späten Mittelalter: Die Auseinandersetzungen zwischen Hohenlohe und Hessen um die Grafschaften Ziegenhain und Nidda. In: WFr 61 (1977), S. 79-110

Kloster Heiningen. In: Wilhelm Kohl u. a. (Hg.): Monasticon Windesheimense. T1.2. Brüssel 1977

Ein Glockenguß in Neuenstein. Ein Bericht, in dem auch von Aberglauben, Preisen und Gewichten die Rede ist. In: Der Haalquell 29 (1977), S. 41-43

Aus der Geschichte der Gemeinde Mainhardt. In: Der Haalquell 29 (1977), S. $57-59$

Von schlechtem Geld und Spesen. In: Der Haalquell 29 (1977), S. 61-62

Aufgaben und Probleme eines standesherrlichen Archivs, dargestellt am Beispiel des Hohenlohe-Zentralarchivs Neuenstein. In: Der Archivar 31 (1978), S. 353-362

Graf Philipp Ernst zu Hohenlohe-Langenburg und sein Schlossbau. In: Ausstellungskatalog Schloß Langenburg. 1978

Zum Einsturz der Augsburger Kirche St. Ulrich und Afra 1474. In: Zs. des Historischen Vereins für Schwaben 72 (1978), S. 122-123

Michael Hospin: Korrekturen an einer Biographie. In: ZWLG 38 (1979), S. 141163

Neue Forschungen zur Baugeschichte von Schloß Langenburg. In: WFr 63 (1979), S. 13-46

Des einen Freud - des andern Leid: Von früheren Ausgrabungen in Mainhardt. In: Der Haalquell 31 (1979), S. 43-44

Von der Entstehung eines Augenscheins: Landkarten als Beweismittel im historischen Gerichtsprozeß. Ein Beispiel aus Hohenlohe. In: BtrLK 1980, 1, S. 9-15

Eine Pestepidemie in der Herrschaft Hohenlohe-Langenburg. In: Schwäbische Heimat 31 (1980), S. 185-187

Aus der Geschichte der Lehensbeziehungen zwischen Würzburg und Hohenlohe. In: WDGB 42 (1980), S. 235-243 
Das Indiz im Hosensack. Eine Episode aus dem 30jährigen Krieg [betr. Raubüberfall auf die Grafen von Hohenlohe durch Soldaten 1646]. In: Der Haalquell 32 (1980), S. 5-8

Über den Augenschein. In: Der Archivar 33 (1980)

Ilshofen in hohenlohischer Zeit. In: Ilshofen. Kleine Stadt an der großen Straße. Ilshofen 1980

Gemeinden im Hohenlohe Kreis. In: Das Land Baden-Württemberg. Bd. IV. Stuttgart 1980

Jus armorum: Ein Wappenstreit zwischen den Fürsten der Linie Hohenlohe-Waldenburg und dem Bischof von Würzburg. In: Herold. Verein für Heraldik, Genealogie und verwandte Wissenschaften 24 (1981), S. 69-88

1000 Jahre Mulfingen? In: WFr 65 (1981), S. 205-209

Zur Provenienz und Datierung der ältesten Gültbücher der Stadt Kirchberg/ Jagst. In: WFr 65 (1981), S. 201-204

Barockbau im Kleinterritorium. In: Volker Himmelein (Hg.): Barock in Baden-Württemberg. Katalog der Ausstellung im Schloß Bruchsal. Bd. 2. Stuttgart 1981

Versuche zur Bildung ständischer Vertretungen in Hohenlohe. In: Von der Ständeversammlung zum demokratischen Parlament: die Geschichte der Volksvertretungen in Baden-Württemberg. Hg. von der Landeszentrale für Politische Bildung Baden-Württemberg. Stuttgart 1982, S. 72-78

Gefährliche Reise zum Lehnherrn: Der Überfall auf die Grafen Hohenlohe. Eine Episode aus dem Dreißigjährigen Krieg. In: BtrLK 1983, 5, S. 12-14

Pockenschutz in Hohenlohe. In: Medizinhistorisches Journal 18 (1983), S. $313-$ 323

Ein Haller Ratsherr verliert sein Amt. Wie David Horlacher über ausgeliehene Akten stolperte. In: Der Haalquell 35 (1983), S. 37-43

Absolutismus in Hohenlohe: Der Fürst als Patriarch. Politik und Administration im 17. und 18. Jahrhundert. In: BtrLK 1983, 6, S. 1-9

Michael Hospin: Humanist, Lehrer, Kartenmaler, 1565-1618. In: Lebensbilder aus Schwaben und Franken 15 (1983), S. 75-85

Die Gründung der ersten deutschen Schule in Ostafrika. In: ZWLG 43 (1984), S. 415-422

Die jüdischen Gemeinden in Baden von der Emanzipation bis 1933. In: Heinz Sproll / Jörg Thierfelder (Hg.): Die Religionsgemeinschaften in Baden-Württemberg. Stuttgart 1984, S. 174-180

Die Zeit der Verfolgung 1933-1945. In: Jael B. Paulus (Hg.): Juden in Baden 1809 bis 1984. 175 Jahre Oberrat der Israeliten Badens. Karlsruhe 1984

Michelbach a. d. Lücke und seine jüdische Gemeinde. In: Schwäbische Heimat 36 (1985), S. 28-36

Teilungen in fränkischen Hochadelshäusern. In: Historischer Atlas von Baden-Württemberg VI.6. Stuttgart 1985

Hohenlohe - Edelherren, Grafen, Fürsten: Territorialentwicklung und Standes- 
erhöhungen im Spiegel ihrer Wappen. In: Gregor Richter (Hg.): Aus der Arbeit des Archivars: Festschrift für Eberhard Gönner. Stuttgart 1986 (= Veröffentlichungen der staatlichen Archivverwaltung Baden-Württemberg), S. 375-405

Juden im deutschen Südwesten. In: Reiner Rinker / Wilfried Setzler (Hg.): Die Geschichte Baden-Württembergs. Stuttgart 1986, S. 188-200

Adelsarchive in Württembergisch Franken. In: Hierzuland - Extra 1, 1987

Die Archive in der Informationsgesellschaft. Überlieferung und archivische Dokumentation im Wandel. Bericht über den deutschen Archivtag. In: Der Archivar 40 (1987)

Hohenlohe und Schlesien. In: Jahrbuch der schlesischen Friedrich-Wilhelms-Universität zu Breslau 29 (1988), S. 199-237

Archive und Wirtschaft. Wirtschaftlichkeit in Archiven (Bericht über den 59. Deutschen Archivtag). In: Der Archivar 41 (1988)

Regensburg und Öhringen. In: WFr 73 (1989), S. 27-44

Aus dem Königreich Württemberg verbannt: Johann Georg Briegel und der Zorn des Monarchen. In: BtrLK 1990, 1, S. 8-13

Archivierung staatlicher medizinischer Unterlagen. In: Der Archivar 43 (1990), S. 390-395

Das Landesarchivgesetz Baden-Württemberg und seine Konsequenzen für die Bewertungsfrage. In: Der Archivar 43 (1990), S. 539-547

Die Aufgaben der Archive im Wandel. Neues Archivrecht - neuartiges Archivgut (Bericht über den 60. Deutschen Archivtag). In: Der Archivar 43 (1990)

Auf dem Wege zur Emanzipation in Württemberg: Stationen der Eingliederung der Schutzjuden in das Königreich im Spiegel der Quellen. Vortrag. In: Verband der Württembergischen Geschichts- und Altertumsvereine / Arbeitskreis für Landes- und Ortsgeschichte 1990, 76, S. 1-9

Hohenlohe. Herrschaft - Grafschaft - Fürstentum. In: Ansichten aus Hohenlohe. Schwäbisch Hall 1990 (= Kataloge des Hällisch-Fränkischen Museums Schwäbisch Hall 4), S. 11-17

700 Jahre Stadt Crailsheim. Crailsheim und die Edelherren von Hohenlohe. In: Beiträge zur Geschichte der Stadt Crailsheim. Zum 700jährigen Stadtjubiläum 1989. Crailsheim 1990 (= Mitteilungsblätter des Crailsheimer Historischen Vereins 6/7)

Hohenlohe - ein geschichtlicher Überblick. In: Otto Bauschert (Hg.): Hohenlohe. Stuttgart 1993 (= Schriften zur politischen Landeskunde Baden-Württembergs 21)

Hohenlohe, Brandenburg-Ansbach, Limpurg. In: Handbuch der baden-württembergischen Geschichte. Bd. 2. Stuttgart 1995, S. 379-388, 401-406, 407-411

Bodo Ebhardt und die Restaurierung von Schloß Neuenstein. In: Harald Siebenmorgen (Hg.): Hofkunst in Hohenlohe: Beiträge einer Arbeitstagung des Badischen Landesmuseums Karlsruhe, des Bildungshauses Kloster Schöntal und 
des Historischen Vereins für Württembergisch Franken. Sigmaringen 1996 (= FoWFr 44), S. 173-208

Hofkunst in Hohenlohe - das historische Umfeld: In: ebd., S. 19-22

Juden in Crailsheim - Familientraditionen - Familientafeln. In: Jüdisches Leben in Crailsheim. Crailsheim 1996

Öffentlichkeitsarbeit - eine Aufgabe der Staatsarchive? In: Archiv und Öffentlichkeit. Stuttgart 1997 (= Werkhefte der staatlichen Archivverwaltung Baden-Württemberg, Reihe A, Heft 9)

Die Kirche im Dorf. In: Peter Schiffer (Hg.): Die Kirche im Dorf. Beiträge einer Arbeitstagung des Württembergischen Landesmuseums Stuttgart, des Bildungshauses Kloster Schöntal und des Historischen Vereins für Württembergisch Franken. Sigmaringen 1998 (= FoWFr 45)

„Unsere unglückliche Unterwerfung unter die württembergische Despotie betreffend“. Die Mediatisierung der hohenlohischen Fürstentümer. In: Volker Himmelein/Hans Ulrich Rudolf (Hg.): Alte Klöster - neue Herren. Bd. 2,2. Ostfildern 2003, S. 883-892

Grabmäler der Hohenlohe. In: Peter Schiffer (Hg.): Zum ewigen Gedächtnis. Beiträge einer Arbeitstagung des Württembergischen Landesmuseums Stuttgart, des Historischen Vereins für Württembergisch Franken, des Bildungshauses des Klosters Schöntal und des Vereins Künstlerfamilie Sommer im Jahr 1999. Stuttgart 2003 (= FoWFr 50), S. 31-42

Ein Augenschein im Kochertal: Ein wichtiger Fund im Staatsarchiv Ludwigsburg. In: Baden-Württemberg / Landesarchivdirektion: Archiv-Nachrichten 26 (2003), S. 12-13

Mediatisierung der hohenzollerischen Fürstentümer. In: Alte Klöster - neue Herren. Die Säkularisation im deutschen Südwesten. Hg. v. Volker Himmelein u. a. 2,2. Ostfildern 2003 , S. $883-892$

Die Territorialentwicklung im württembergischen Nordosten in der Napoleonzeit. In: Karl-Heinz Wüstner (Hg.): Soldatenehr als Möbelzier. Uniformierte auf Möbeln und anderen Objekten der Volkskunst; Begleitbuch und Katalog zur gleichnamigen Sonderausstellung im Rößler-Museum Untermünkheim vom 18. Mai bis 20. Juli 2003. Untermünkheim 2003, S. 61-64

Öhringen: Hohenlohes heimliche Hauptstadt. In: Hans-Georg Wehling (Hg.): Wegmarken südwestdeutscher Geschichte. Stuttgart 2004, S. 198-207

Ein Totschlag auf dem Halberg und seine Folgen oder die Entstehung der ältesten Ortsansicht von Niedernhall. In: WFr 90/91 (2006/07), S. 131-142

Revolutionäre in Waldenburg? Unruhen am Ende des Alten Reichs. In: WFr 93 (2009), S. 7-29

Der Baumeister Bernhard Sporer und sein letztes Bauwerk: die St.-Anna-Kapelle in Öhringen. In: ZWLG 68 (2009), S. 157-183

Wendel Hipler (um 1465-1526). In: Gesellschaft für Fränkische Geschichte: Veröffentlichungen der Gesellschaft für Fränkische Geschichte. Reihe 7.A, Fränkische Lebensbilder 22 (2009), S. 65-78 
Otto May und Reinhold Mayer - eine Freundschaft in der Bewährung. In: ZWLG 69 (2010), S. 351-385

Staat, Kirchen und Finanzen - die St.-Anna-Kapelle in Öhringen nach der Mediatisierung. In: WFr 94 (2010), S. 209-219

Identitätsstiftung durch historische Information: Hohenlohe und die Archive seiner Herrschaft. In: Robert Kretzschmar (Hg.): Staatliche Archive als landeskundliche Kompetenzzentren in Geschichte und Gegenwart: zum 65. Geburtstag von Volker Rödel. Stuttgart 2010, S. 181-197

Zwischen Widerstand und Gestapo: Dr. Hugo Bühler, Abwehrbeauftragter der Firma Bosch in Stuttgart. In: ZWLG 70 (2011), S. 455-488

Kleinterritorium und Standeserhöhung im Alten Reich, die Erhebung des Hauses Hohenlohe in den Reichsfürstenstand. In: Historisches Jahrbuch 132 (2012), S. $177-219$

Hohenlohe: Haus und Land im Reich der Burgen und Schlösser. In: Reinhold Weber (Hg.): Baden-Württembergische Erinnerungsorte. Stuttgart 2012, S. 110-119

Die hohenlohische Erbeinung von 1511. In: Peter Schiffer (Hg.): Aufbruch in die Neuzeit. Das nördliche Württemberg im 19. Jahrhundert. Ostfildern 2012 (= FoWFr 53), S. 113-119

Der Lehnsadel der Hohenlohe in der Verschuldungskrise des Spätmittelalters. In: Niederadel um Crailsheim. Crailsheim 2012

\section{Festschriften}

Festschrift für Gerhard Taddey (= WFr 86, 2002) [Jahrbuch des Historischen Vereins für Württembergisch Franken]

Hg. Norbert Hofmann: Archivisches Arbeiten im Umbruch: Vorträge des Kolloquiums der staatlichen Archivverwaltung Baden-Württemberg am 26. und 27.11.2002 im Staatsarchiv Ludwigsburg aus Anlass der Verabschiedung von Herrn Professor Dr. Gerhard Taddey. Stuttgart 2004

\section{Sonstiges}

Langenburg. 750 Jahre Schloss und Stadt. Langenburg 1976

Niedernhall und sein Rathaus - Festschrift zur Rathauseinweihung. Niedernhall 1984

Texte und Motive („Drehbuch“) zum Dokumentarfilm: Hohenlohe - aus der Geschichte einer Landschaft (ARD, Landes- und Kreisbildstellen) 1984

(Gemeinsam mit Otto Hesler): Stadtkirche Neuenstein. Neuenstein 1986

Vorformen des Investmentsparens. In: 150 Jahre individueller Dienst am Kunden. Heilbronn 1987, S. 81-86

Bearb.: Schloss Neuenstein und sein Museum. Von Constantin Prinz zu Hohenlohe. München ${ }^{3} 1990$ (= Große Baudenkmäler 155) 
Texte und Motive („Drehbuch“) zum Dokumentarfilm: Niedernhall, Geschichte der Stadt, 1990

Texte und Motive („Drehbuch“) zum Dokumentarfilm: Der Hohenlohekreis, 1993

Bearb.: Schloss Neuenstein und sein Museum. Von Constantin Prinz zu Hohenlohe. München ${ }^{4} 1995$ (= Große Baudenkmäler 155)

Bearb.: Schloss Neuenstein und sein Museum. Von Constantin Prinz zu Hohenlohe. München ${ }^{5} 2001$ (= DKV-Kunstführer 155)

Schwäbisch Hall: Von der Stadt des Salzes zur Stadt der Bausparkasse. In: Wegmarken südwestdeutscher Geschichte. Stuttgart 2004, S. 220-229

Unter drei Landesherren: Die Herrschaft Hohenlohe-Kirchberg 1806-1810 [Text, der anlässlich eines Vortrags entstanden ist; der Vortrag wurde am 22.10.2010 in der Kirchberger Festhalle gehalten.] Kirchberg/Jagst 2010 (= Kirchberger Hefte 11)

Redaktion der Quellenbeilagen in den „Archivnachrichten“ Nr. 1-6

Außerdem wurden seit 1965 zahlreiche Findbücher mit wissenschaftlichen Einleitungen im Hohenlohe-Zentralarchiv Neuenstein, im Hauptstaatsarchiv Stuttgart und im Staatsarchiv Ludwigsburg erstellt. Nicht erfasst sind auch kleinere Veröffentlichungen in „Hohenloher Leben“, der Beilage zur Hohenloher Zeitung und die zahlreichen Buchbesprechungen in WFr, ZWLG, ZGO, Der Archivar, den Blättern für Landesgeschichte usw.

Daneben hat Gerhard Taddey zahlreiche öffentliche Vorträge in historischen Vereinen, Volkshochschulen, bei Fachveranstaltungen, bei festlichen Anlässen und Jubiläen, im Rundfunk usw. gehalten und wissenschaftliche Exkursionen geleitet, v. a. in Hohenlohe. 